\title{
Transhumanismo en la ciencia ficción: exégesis de la saga «Fundación» de Isaac Asimov
}

\author{
Science Fiction Transhumanism: \\ Exegesis of the Foundation saga by Isaac Asimov
}

JOSÉ LUIS ARROYO BARRIGÜETE

UNIVERSIDAD DE SEVILLA

Artículo recibido el / Article received: 2017-06-14

Artículo aceptado el / Article accepted: 2018-01-29

RESUMEN: Este artículo analiza varias de las corrientes transhumanistas, como el singularitarianismo, inmortalismo, posgenerismo y abolicionismo, en la saga de la Fundación de Isaac Asimov. Partiendo de los quince libros que conforman dicha saga, se examina la visión de Asimov sobre estas ideas, y muestra cómo las diferentes novelas tratan sus complejas implicaciones, ya que contrariamente a la interpretación más habitual, el autor fue mucho más allá de sus historias de robots al tratar temáticas transhumanistas.

Palabras clave: Transhumanismo, singularitarianismo, inmortalismo, posgenerismo, abolicionismo, ciencia ficción, Isaac Asimov y saga de la Fundación.

ABSTRACT: This article analyzes several transhumanist currents, like singularitarianism, immortalism, postgenderism and abolitionism, in the Foundation series of Isaac Asimov. On the basis of the 15 books that make up the saga, Asimov's vision of these ideas is examined to show how these novels address their complex implications, because contrary to the usual interpretation, the author went far beyond his robot stories for dealing with transhumanist topics.

Keywords: Transhumanism, singularitarianism, immortalism, postgenderism, abolitionism, science fiction, Isaac Asimov and Foundation series. 


\section{INTRODUCCIÓN}

El término transhumanismo parece que fue usado por primera vez en 1927 por Julian Huxley, biólogo fundador del Fondo Mundial para la Naturaleza, y que fue también el primer director general de la UNESCO (Bostrom, 2005 y 2011). Su planteamiento general es la modificación del ser humano mediante la tecnología, actuando como estadio intermedio entre el humano y el poshumano (González Melado y Martínez Guisasola, 2012). Como señala Marín (2012), a diferencia de la tradición prometeica, que busca un perfeccionamiento del cuerpo humano pero respetando ciertos límites impuestos por su propia naturaleza, el transhumanismo representa una corriente de pensamiento firmemente asentada en la tradición fáustica, que busca superar todas las limitaciones derivadas del carácter material del cuerpo humano, ignorando cualquier barrera. Sin embargo, sería ingenuo asumir que se trata de un movimiento homogéneo, pues existen numerosas variantes dentro del transhumanismo con notables diferencias entre ellas, dependiendo tanto del enfoque en el uso de la tecnología como en la finalidad última de esta; desde las propuestas más obvias como el inmortalismo, que propugna la consecución de un ser humano inmortal, hasta el posgenerismo, que defiende la abolición del género sexual mediante técnicas de ingeniería genética.

Al margen de las reflexiones filosóficas sobre sus implicaciones, se trata de una propuesta intelectualmente estimulante y, por tanto, no resulta extraño que la literatura basada en el extrañamiento cognitivo, tal y como la definió Suvin (1984), la haya incorporado con pasión a sus tramas. Definir el género de la ciencia ficción (CF) no es en absoluto sencillo, debido principalmente a «la inexistencia de límites precisos para la temática y los enfoques que utiliza» (Barceló, 1990: 24), pero sea cual sea su definición, un concepto como el transhumanismo incorpora todos los elementos para resultar interesante en este contexto. No es de extrañar que la CF haya explorado exhaustivamente las posibilidades, consecuencias e implicaciones de las propuestas transhumanistas. De este modo encontramos desde interpretaciones muy directas, como las del ciberpunk, que suele ambientarse en un distópico futuro posindustrial y propone una evolución basada en diversas suertes de fusión hombre-máquina, hasta sutiles e inquietantes posibilidades, como las planteadas en El fin de la infancia (Clarke, 1953).

Isaac Asimov es probablemente uno de los escritores de CF que con mayor frecuencia se menciona al hablar de transhumanismo ${ }^{1}$ (véase por ejemplo

1. Los transhumanistas frecuentemente han encontrado su inspiración en la literatura de ciencia ficción. Sin embargo, como apunta Burdett (2015: 68), «one can't help but notice that science fiction literature is treated like the younger, less serious brother of contemporary transhumanists. [...] In their attempts to legitimise their movement they are often found to actually distance themselves from science fiction». 
Bostrom, 2005; Tirosh-Samuelson, 2007; Babich, 2013; Sandu y Caras, 2013; Ali Mirenayat et al., 2017), pero paradójicamente se suele hacer respecto a sus relatos sobre robots, lo que resulta extraño teniendo en consideración que otras obras suyas profundizaron sustancialmente más en varias de las sutilezas de esta corriente de pensamiento. Como analizaremos a continuación, Asimov trató en profundidad varias de sus ideas y, contrariamente, a lo que algunos transhumanistas sostienen, sus conclusiones no fueron precisamente positivas.

\section{UNA APROXIMACIÓN A LA SAGA DE LA FUNDACIÓN}

Desde una perspectiva holística podemos considerar que la saga de la Fundación está compuesta por un conjunto de quince libros (Asimov, 1950a, 1950b, 1951a, 1951b, 1952a, 1952b, 1953, 1954, 1957, 1982, 1983, 1985, 1986, 1988, 1993) más otros tres que no fueron escritos por el propio Isaac Asimov (Bear, 1998; Benford, 1997; Brin, 1999), sino por otros escritores tras su fallecimiento. En ocasiones se incluye también la novela Crisis psicohistórica (Kingsbury, 2001), inspirada en el universo asimoviano pero que modifica los nombres originales por no haber sido aprobada por sus herederos. Existen también relatos cortos, apócrifos en su mayoría, que habitualmente no se consideran como parte de la saga.

Centrándonos en los escritos del propio Asimov, es preciso destacar al menos cuatro aspectos de esta saga, considerada junto con Dune, de Frank Herbert, una de las más importantes de la CF. La primera es la gran heterogeneidad de sus trabajos, en los que encontramos desde el relato corto, como en Yo robot», que no es sino un compendio de estos, hasta las extensas novelas que conforman los últimos capítulos de la saga. La segunda característica reseñable es que, al ser escrita a lo largo de un período de más de cuarenta años, se aprecia claramente la evolución del autor, tanto en su estilo literario como en sus ideas y reflexiones, cuando crea un universo más y más complejo que además no siempre resulta coherente. En tercer lugar, existe una fuerte intertextualidad con textos científicos o históricos. De hecho, la formación científica de Asimov le permitió, a lo largo de toda su obra, incluir ciertos elementos que actuasen cono gancho para el lector con ciertos conocimientos en este campo. Un ejemplo, ajeno a la Fundación pero quizá de los más interesantes y sutiles, lo encontramos en El fin de la eternidad (Asimov, 1955). En la novela se describe con cierto detalle cómo fue descubierto, de forma puramente empírica, el campo temporal, que sin embargo no pudo ser mejorado tras el descubrimiento posterior de sus fundamentos teóricos. Es inevitable plantearse en qué medida Asimov está haciendo aquí una alusión al desarrollo de la termodinámica; si bien en 1816 Robert Stirling patentó su motor basado en un ciclo termodinámico, no fue hasta 1824, con la publicación de la 
única obra de Nicolas Léonard Sadi Carnot, que se pudieron explicar los fundamentos teóricos de su invención. Adicionalmente el motor Stirling es el único que presenta un rendimiento (teórico) que alcanza el máximo posible, determinado por el rendimiento del motor ideal de Carnot; motores desarrollados con posterioridad sobre la base de otros ciclos, no alcanzan dicho límite teórico.

Precisamente, como consecuencia de esta sólida formación científica, Asimov no tuvo reparo alguno en describir las características de las ecuaciones que componían el mosaico de la psicohistoria, el campo temporal y otras de las teorías que imaginó en sus novelas, combinando dichas descripciones con explicaciones verbales de tipo filosófico. En el caso concreto de la psicohistoria, llega incluso a rozar la écfrasis al describir de una manera un tanto poética el funcionamiento del primer radiante, objeto que permitiría la representación tridimensional de las ecuaciones que conforman la psicohistoria. Sin embargo, el abuso de los diálogos como base narrativa, estilema del autor, es también la tónica dominante a la hora de explicar los detalles de sus teorías. Debido a ello la sensación de artificiosidad suele ser elevada, ya que no resulta sencillo crear situaciones en las que una explicación de la teoría esté justificada, máxime cuando dicha explicación ha de ser para no iniciados, a fin de que el lector pueda comprenderla. Esto obliga a Asimov a forzar conversaciones entre un especialista y un profano, o en el mejor de los casos un diletante, e incluso a buscar diálogos poco creíbles en los que dos especialistas debaten en términos metafóricos en lugar de recurrir a la precisión del lenguaje matemático que ambos podrían emplear. Como complemento a este tipo de explicaciones, el autor recurre en ocasiones a elementos paratextuales, principalmente en modo de referencias a la Enciclopedia galáctica al inicio de los capítulos de la saga de la Fundación. Sin embargo, dichas descripciones apenas sirven para matizar determinados elementos; el peso de la descripción recae en los diálogos.

Por último, una reseñable característica de esta saga, y de la obra de Asimov en general, es que requiere al lector un mínimo esfuerzo para acceder a su mundo ficcional, ya que al igual que sucede con otras obras de CF (véase por ejemplo el análisis de las sagas cinematográficas Alien y Predator de Catalan, 2010), incorpora pocos elementos realmente extraños a la realidad cotidiana. Esto ha sido criticado en ocasiones, y se ha acusado a Asimov de describir un futuro excesivamente parecido al presente.

En relación con sus reflexiones sobre las ideas transhumanistas, es necesario indicar que gran parte de estas se desarrollaron formalmente en los años ochenta y noventa, coincidiendo con las últimas obras del autor. Es cierto que las semillas ya estaban sembradas desde mucho antes, pero como señala Duarte (2010: 55)

en los planteamientos iniciales de Asimov [...] se trasluce una ingenua presunción sobre la culminación de la condición humana y de su evolución como 
especie. Loable al fin y al cabo en cuanto a las mejoras médicas o científicas que se puedan derivar, pero en definitiva no más que de lo mismo.

\section{SINGULARITARIANISMO Y EVOLUCIÓN TECNOLÓGICA}

El concepto de singularidad puede entenderse como el surgimiento de una inteligencia sobrehumana que acelerará el progreso tecnológico y social de un modo tal que resulta imposible de predecir antes de su aparición. Si bien esta idea ya se había utilizado con anterioridad, fue Vernor Vinge quien la populariza en su novela La guerra de la paz (Vinge, 1984), y posteriormente en su ensayo sobre la llegada de la singularidad tecnológica (Vinge, 1993) de manera que el concepto ha quedado así establecido:

The acceleration of technological progress has been the central feature of this century. We are on the edge of change comparable to the rise of human life on Earth. The precise cause of this change is the imminent creation by technology of entities with greater-than-human intelligence. [...] When greater-than-human intelligence drives progress, that progress will be much more rapid. [...]And what happens a month or two (or a day or two) after that? I have only analogies to point to: The rise of humankind. We will be in the Post-Human era» (Vinge, 1993: 1-4)

La conocida Singularity University, que tanta repercusión mediática ha tenido en parte por sus planteamientos filosóficos, en parte por su metodología de estudio, basa su denominación en este concepto. Realmente el término singularidad hace referencia al concepto matemático del mismo nombre, esto es, una región en la que una función matemática presenta comportamientos inesperados. En el ámbito de la física, y más concretamente en teoría de la relatividad general, este vocablo presenta un significado similar, y se refiere a un punto del espaciotiempo en el que la gravedad se hace infinita, $\mathrm{y}$, por tanto, es imposible predecir el comportamiento de las leyes físicas en ese punto.

Los defensores del singularitarianismo plantean que los avances científicos en ramas como la computación, la nanotecnología, la robótica, la genética y la neurología permitirán el surgimiento de esta singularidad, y que debemos trabajar para que se produzca lo antes posible y del modo más seguro posible para el ser humano, de manera que como resultado se logre un desarrollo y evolución de este. En la medida en que estas ideas consideran la transformación del ser humano como la consecuencia última, el singularitarianismo encuentra importantes puntos comunes con el transhumanismo más convencional. 
Pese a que desde el punto de vista de la CF este concepto resulta sumamente atractivo, Asimov, en su saga de la Fundación, no plantea esta posibilidad en ningún momento, es decir, la aparición de robots positrónicos no genera una disrupción en el progreso científico de la humanidad. Muy al contrario, el hilo conductor de la saga Fundación es la denominada psicohistoria, ${ }^{2}$ cuya existencia exige forzosamente que tal singularidad no se dé, como se analizará a continuación.

La primera de las novelas de esta saga, comienza con una descripción de la ciencia psicohistórica que resulta bastante ilustrativa: una rama de las matemáticas que trata sobre las reacciones de conglomeraciones humanas ante determinados estímulos sociales y económicos. Si bien esta definición se fue puliendo tanto en las secuelas como en las precuelas que Asimov publicó a lo largo de los años, no sufrió en esencia modificaciones sustanciales. Así, de acuerdo con el planteamiento del autor, la psicohistoria permitiría predecir en términos estadísticos el futuro de una sociedad galáctica en la que la humanidad se hallaba diseminada por cerca de veinticinco millones de planetas. El desarrollo de esta ciencia desde su embrión inicial hasta su máximo desarrollo, así como el crecimiento aparentemente inexorable de la Fundación hacia el Segundo Imperio Galáctico, constituyen el hilo conductor de todas las novelas de la saga. Predecir el futuro deja por tanto de ser un fenómeno paranormal para erigirse como una rama de las matemáticas, que lógicamente implica un poder incalculable basado en el conocimiento científico, y sustituye así a la potencia militar o los poderes sobrenaturales. Realmente, y en especial en las últimas entregas de la saga, la psicohistoria debe ser interpretada más como un constructo social del universo asimoviano que como una verdadera fuerza motriz independiente, lo que de hecho desvirtúa sensiblemente el espíritu de las primeras novelas.

Visto desde la perspectiva actual, Asimov estaba describiendo una versión extrema de la cliodinámica, disciplina científica relativamente reciente y que pretende algo similar: transformar la historia en una ciencia analítica (Turchin, 2008). Habitualmente se considera como objetivo principal de dicha ciencia, llegar a tener la capacidad de calcular las consecuencias de determinadas elecciones, con la finalidad de favorecer el desarrollo de sistemas sociales y evitar las posibles consecuencias negativas. Sin embargo algunos especialistas insisten en las posibilidades predictivas de esta disciplina: «An analytical, predictive history, or cliodynamics, as I propose we call it, is eminently possible» (Turchin, 2011: 169).

2. Es necesario mencionar que existe una disciplina real, denominada psicohistoria, que definiéndose como la ciencia de las motivaciones históricas, trata de comprender desde un enfoque interdisciplinar el origen emocional de los movimientos sociales y políticos. No obstante, el término psicohistoria en la obra de Asimov tiene un sentido diferente. 
Asimov incorporó a sus teorías imaginarias elementos que las dotasen de cierta verosimilitud. En este sentido, y siguiendo el esquema habitual en la formulación de teorías físicas, planteó una serie de postulados sobre los que fundamentar la psicohistoria. De hecho, en este caso todo el cuerpo teórico se fundamenta en dos premisas esenciales: ${ }^{3}$ trabajar con un número suficientemente grande de individuos y que el conjunto de la humanidad desconozca el análisis psicohistórico, a fin de que su reacción sea verdaderamente casual. Ambas hipótesis son, en realidad, una transcripción casi literal de dos de los postulados básicos de la teoría cinética de los gases, ${ }^{4}$ y no es algo casual: como el propio autor manifiesta por boca de algunos de los personajes de su obra, Hari Seldon desarrolló la psicohistoria tomando como modelo dicha teoría. Como se ha señalado en incontables ocasiones, el enfoque asimoviano de la cliodinámica peca de un reduccionismo casi ingenuo, muy próximo al determinismo de Laplace, y es quizá en este paralelismo con la teoría cinética de los gases donde se puede observar con mayor nitidez. La comparación, aunque solo sea en términos metafóricos, de moléculas individuales de gas con seres humanos no se sostiene desde ningún punto de vista, y roza la conocida «falacia de la falsa analogía». Basta considerar la capacidad cognitiva de los individuos para comprender que no es solo una cuestión de escala en lo que a complejidad se refiere; estamos hablando de fenómenos muy diferentes que dudosamente pueden tener relación alguna. En este sentido, probablemente el principal problema de Asimov fue desconocer lo que hoy denominamos teoría del caos, aunque desde luego no se trata de un error suyo: los descubrimientos de Edgard Lorenz que dieron lugar al desarrollo de dicha teoría se produjeron a principios de los sesenta, años después de la publicación de la primera novela de la Fundación. Probablemente si Asimov la hubiese conocido, habría comprendido la futilidad de intentar desarrollar una cliodinámica tan extrema como la que pretende ser la psicohistoria.

En todo caso, y al margen de las críticas que podamos hacer desde la perspectiva científica actual, la propia existencia de la psicohistoria asimoviana niega la posibilidad de una singularidad que haga imprevisible el desarrollo tecnológico posterior. Bien es cierto que en el momento de escribir sus novelas el concepto de singularidad aún no había sido convenientemente desarrollado, pero aun así queda clara la postura del autor en este sentido: frente a un evento disruptivo como el

3. En Fundación y tierra (Asimov, 1986), una de las últimas novelas de la saga, se añade una premisa adicional que la psicohistoria asume de forma implícita: los seres humanos son la única raza inteligente.

4. De los postulados de que parte la teoría cinética de los gases (véase por ejemplo Fernández Ferres y Pujal Carrera, 1996: 355) merece la pena destacar dos ideas: la necesidad de trabajar con un número grande de moléculas, y que estas se comporten de un modo aleatorio pero respetando las leyes de la dinámica. 
que se postula desde el singularitarianismo, Asimov concebía la evolución científica y tecnológica como un proceso continuo y relativamente parsimonioso.

\section{INMORTALISMO Y DECADENCIA}

El inmortalismo parte de la creencia de que es posible, y deseable, prolongar la vida de una manera drástica gracias a la tecnología. Existen otras corrientes, derivadas de esta idea, como el trascendentismo, pero en última instancia todas comparten como denominador común la búsqueda de la emortalidad o incluso la inmortalidad. Probablemente en la obra de Asimov en la que más nítidamente se aprecia su opinión a este respecto sea en Los robots del amanecer (Asimov, 1983), cuarta novela correspondiente al denominado «Ciclo de la Tierra» y que por su fecha de escritura, más de treinta años después de la primera novela de la saga, recoge un conjunto de reflexiones más profundas que las que pueden apreciarse en sus primeros escritos.

En relación con el inmortalismo, dos son las principales objeciones que plantea Asimov. La primera aborda directamente el inmovilismo inherente a una sociedad excesivamente longeva, derivado de su apego a una vida larga y en general agradable, que generaría una importante aversión al riesgo. Según Asimov, el binomio riesgo- rentabilidad que estas sociedades estarían dispuestas a asumir indefectiblemente se sesgaría hacia las opciones más conservadoras, y limitarían por tanto sus posibilidades de éxito, materializadas en el caso de la novela en el proceso de colonización espacial:

Los terrícolas tienen una vida corta y están acostumbrados a reponer rápidamente las que se pierden. Carecen de nuestro respeto y consideración por la vida humana individual. Por eso se esparcirán por los nuevos mundos a toda costa, multiplicándose como insectos, y se apropiarán de toda la galaxia mientras nosotros [seres humanos longevos] estemos todavía dando los primeros pasos. (Asimov [1983]2008: 403)

La segunda gran objeción, íntimamente relacionada con la primera, afronta las consecuencias para el progreso científico de la excesiva longevidad. En este caso, y visto con la perspectiva actual, la reflexión es un tanto ingenua, al asumir la investigación como un proceso individual. Hoy sabemos que dado el volumen de conocimiento científico y tecnológico disponible, así como su complejidad, no es viable el progreso sin una íntima colaboración de decenas de científicos, en muchos casos de diferentes campos. Su visión individualista responde a su experiencia personal en un momento histórico en el que las circunstancias eran muy diferentes. 
Las presiones en una sociedad longeva son menores. Aquí, los científicos disponen de tres siglos o más para dedicarse a un problema, por lo que se piensa que un trabajador en solitario puede conseguir en ese período de tiempo progresos significativos en su campo concreto. Así se hace posible sentir una especie de avaricia intelectual, de ansia por conseguir algo por uno mismo o de asumir el derecho de propiedad sobre una faceta concreta del progreso. El científico puede desear entonces que el progreso general adquiera un ritmo más pausado, antes que ofrecer a la comunidad lo que concibe como asunto propio y de su exclusiva propiedad. Como resultado de esa manera de pensar, el progreso general presenta una considerable ralentización en los mundos espaciales, hasta el punto de resultar difícil seguir el ritmo del trabajo efectuado en la Tierra, pese a nuestras enormes ventajas sobre su planeta. (Asimov [1983]2008: 222)

Curiosamente, apenas sí le da relevancia a uno de los problemas que más frecuentemente se utilizan como argumento contra en inmortalismo, y que no es otro que la necesidad de un estricto sistema de control de la natalidad a fin de evitar un crecimiento malthusiano de la población. En la novela se menciona, apenas de pasada, la existencia de ciertas restricciones al número de hijos que un individuo puede llegar a tener, aunque sin profundizar en las consecuencias sociales que tal política podría derivar.

Sus dos principales objeciones podrían ser rebatidas desde un punto de vista filosófico, en tanto que parte de algunas premisas cuestionables, como el hecho de que los individuos que componen las sociedades longevas no incluyen elementos disfuncionales. El simple aburrimiento podría materializarse en un deseo de riesgo que, de afectar a un número relevante de individuos, dotaría de un cierto impulso vital al conjunto de la sociedad. Y como ya se ha comentado, su planteamiento sobre el progreso científico es, visto con la perspectiva actual, un tanto ingenuo. Sin embargo, y al margen de la valoración que pueda hacerse de sus hipótesis, queda claro que Asimov intuía importantes problemas en el concepto de inmortalismo, hasta el punto de que en esta pugna más o menos velada entre humanos de vida corta y humanos longevos por el control de la galaxia, son los primeros quienes finalmente se alzan con la victoria en posteriores novelas de la saga.

\section{POSGENERISMO Y HUMANIDAD}

Una buena descripción de qué puede entenderse por posgenerismo la encontramos en Dvorsky y Hughes (2008: 1), quienes lo apuntan como una de las posibilidades que podría ofrecer la modificación del ser humano que propugna el transhumanismo: 
Postgenderists argue that gender is an arbitrary and unnecessary limitation on human potential, and foresee the elimination of involuntary biological and psychological gendering in the human species through the application of neurotechnology, biotechnology and reproductive technology»

En su novela Fundación y tierra, Asimov (1986) aborda el posgenerismo de una manera muy directa, imaginando un planeta completo, Solaria, poblado por individuos que han optado por abolir los géneros sexuales mediante la ingeniería genética. Se trataría por tanto de la versión más radical del posgenerismo, en tanto que la interpretación mayoritaria de esta corriente de pensamiento no pasa tanto por una eliminación de los géneros sexuales, como por la libre elección de estos por parte de cada individuo.

Los solarianos, que se definen a sí mismos como «humanos totales», muestran una actitud desdeñosa hacia los «medio humanos» que solo cuentan con un sexo, considerándolos poco más que animales. De hecho, los robots sirvientes solarianos ni siquiera sufren las restricciones de la primera ley de la robótica ${ }^{5}$ al tratar con ellos, pues de acuerdo con sus patrones, únicamente los humanos totales, con los dos sexos, entrarían en la categoría de humanos.

Las razones que esgrimen los solarianos para su transformación son algo diferentes a los que plantean los partidarios del posgenerismo, aunque en última instancia en ambos casos se plantea como un modo de superar ciertos problemas, llámese libertad en el primer caso, llámese igualdad en el segundo.

La postura de Asimov con respecto a Solaria es clara: pone en boca de los protagonistas de la novela duras críticas al sistema social basado en el aislamiento que existe en el planeta. Pero se trata de críticas centradas en dicho aislamiento, no así en las características físicas de sus habitantes. No es sino al final de esta obra cuando se entrevé su opinión sobre este tipo de alteración física, al poner en duda que los solarianos puedan seguir considerándose humanos debido a que los cambios genéticos autoinducidos tanto en el ámbito sexual como en otros.

En toda la Historia humana, ninguna otra inteligencia nos ha amenazado, que nosotros sepamos. Bastaría con que esto continuase durante unos pocos siglos, tal vez poco más de una milésima del tiempo que llevamos de civilización, para que estuviésemos a salvo. A fin de cuentas -y aquí sintió Trevize una súbita aprensión que se obligó a pasar por alto- no es como si ya tuviésemos al enemigo entre nosotros. Y no bajó la mirada para no encontrarse con los ojos reflexivos de Fallom (hermafrodita, transductora, diferente) que le estaban mirando, fijos, insondables. (Asimov [1986]1992: 523-524)

5. Un robot no dañará a un ser humano ni permitirá por inacción que sufra daño. 


\section{ABOLICIONISMO Y MADUREZ}

La corriente de pensamiento abolicionista plantea ese uso de la tecnología para eliminar el sufrimiento humano, tanto en su vertiente física como psicológica. Si bien es cierto que el abolicionismo suele estar referido al uso de la bioetecnología, Asimov abordó esta problemática de un modo diferente: planteó el uso de robots como vía para lograr reducir el sufrimiento del ser humano, liberándole de las tareas tediosas y peligrosas al tiempo que velaban por su seguridad y felicidad. Se trata por tanto de un planteamiento precursor, más tibio, de lo que actualmente identificamos con el proyecto abolicionista, pero con muchos elementos comunes.

Cuando Asimov elaboró con John Campbell las tres ${ }^{6}$ leyes de la robótica (Cuadros Contreras, 2008), las planteó como un mecanismo de salvaguarda para los seres humanos, así como la excusa para muchas de sus tramas tanto en sus relatos cortos como novelas, y en ellas se evidencia la prioridad absoluta de velar por el bien de los seres humanos. De hecho, en uno de los relatos cortos recogidos en Yo, robot (Embustero en Asimov, 1950a) se plantea que el concepto de evitar el daño a un ser humano debe entenderse en un sentido amplio, incluyendo sin duda el daño psicológico.

Asimov, a medida que avanza en la construcción de su saga, va planteando cada vez más objeciones a las consecuencias que una relación humano-robot de este tipo provocaría en la sociedad. Son numerosas sus alusiones a la decadencia de una sociedad mimada y sobreprotegida por sus asistentes robots, custodios permanentes del bienestar de sus individuos. E incluso llega a plantear que esta relación acabaría provocando resentimiento en los seres humanos, haciendo que finalmente acabasen rechazándolos: «Los nuevos mundos fueron fundados con otros criterios y los fundadores no quisieron ni recordar su amarga humillación de niños sometidos a niñeras-robots» (Asimov [1982]1997: 406). Parece por tanto que el pensamiento de Asimov fue evolucionando en el sentido de encontrar cada vez más objeciones a la sobreprotección del ser humano.

6. Un robot no dañará a un ser humano ni permitirá por inacción que sufra daño. 2. Un robot debe obedecer las órdenes dadas por los seres humanos, excepto si estas órdenes entrasen en conflicto con la primera ley. 3. Un robot debe proteger su propia existencia siempre que esta protección no entre en conflicto con la primera o la segunda ley. Posteriormente, Asimov añadiría una cuarta ley, la ley cero, con prioridad sobre las tres anteriores: un robot no dañará a la humanidad ni permitirá por inacción que esta sufra daño. Las implicaciones filosóficas de esta última ley, así como las dificultades para su implementación en situaciones concretas son un importante elemento en varias de las obras de Asimov 


\section{CONCLUSIONES}

Como hemos visto, al final de la saga de la Fundación muchas de las ideas transhumanistas habían sido analizadas y criticadas, en algunos casos con dureza, y apenas una resistió el ataque de Asimov. El tecnogaianismo, que postula emplear la tecnología para ayudar a restablecer el medio ambiente terrestre, fue tratado en las dos últimas novelas de su saga, Los límites de la Fundación y Fundación y tierra, aunque añadiendo un importante elemento adicional: la creación de una gran conciencia colectiva a escala planetaria como fusión de todos los organismos vivos y elementos inertes del planeta. Sin embargo, el aspecto relevante desde el punto de vista del tecnogaianismo, es que Asimov concibe esta transformación de planeta inerte a planeta vivo como un proceso en el que la tecnología juega un papel esencial, como no podría ser de otra manera, tecnología materializada en forma de robots que ponen en marcha el proceso de transformación y posteriormente supervisan su evolución. También insiste el autor en el magnífico equilibrio ecológico alcanzado en Gaia -lo es justamente la base de los postulados tecnogaianistas- siempre bajo la supervisión y control de un reducido número de robots cuya misión sería precisamente velar por el correcto funcionamiento del sistema planetario.

Parece por tanto injustificado que se considere a Asimov como adalid del transhumanismo, y difícilmente puede llegarse a esta conclusión. La exégesis de la que probablemente es su principal obra de CF no deja lugar a dudas de un posicionamiento claramente contrario a los postulados transhumanistas, tanto generales como específicos de sus distintas corrientes. Es posible disentir de sus conclusiones, y puede argumentarse que adolece de rigor en la reflexión, lo que en ciertos casos es objetivamente cierto debido al momento en que se escribieron sus obras, cuando aún no se disponía de los conocimientos científicos, sociológicos y filosóficos que tenemos hoy. Pero aun así, esa era su opinión, y pocas dudas quedan de que su apuesta pasaba por los humanos sexuados, autónomos y de vida corta.

\section{REFERENCIAS BIBLIOGRÁFICAS}

Ali Mirenayat, S.; Baizura Bahar, I.; Talif, R. Mani, M. (2017): «Science Fiction and Future Human: Cyborg, Transhuman and Posthuman», Research Result. Theoretical and Applied Linguistics, 3(1), 76-81.

Asimov, I. (1950a): I, Robot, EE.UU.: Gnome Press.

- (1950b): Pebble in the Sky, EE.UU.: Doubleday.

- (1951a): The Stars, Like Dust, EE.UU.: Doubleday.

- (1951b): Foundation, EE.UU.: Doubleday. 
- (1952a): Foundation and empire, EE.UU.: Doubleday.

- (1952b): The Currents of Space, EE.UU.: Doubleday.

- (1953): Second foundation, EE.UU.: Doubleday.

- (1954): The Caves of Steel, EE.UU.: Doubleday.

- (1955): The End of Eternity, EE.UU.: Doubleday.

- (1957): The Naked Sun, EE.UU.: Doubleday.

- ([1982] 1997): Foundation's Edge, EE.UU.: Doubleday. Edición citada: Los Límites de la Fundación. Trad. M. T. Segur. Barcelona: Plaza y Janes.

- ([1983] 2008): The Robots of Dawn. EE.UU.: Doubleday. Edición citada: Los Robots del Amanecer. Trad. M. T. Segur y H. Sabaté. Barcelona: Debolsillo.

- (1985): Robots and Empire, EE.UU.: Doubleday.

- ([1986] 1992): Foundation and Earth, EE.UU.: Doubleday. Edición citada: Fundación y Tierra. Trad. J. Ferrer Aleu. Barcelona: Plaza y Janes.

- (1988): Prelude to Foundation, EE.UU.: Doubleday.

- (1993): Forward the Foundation, EE.UU.: Doubleday.

BARCELó, M. (1990): Ciencia ficción: guía de lectura, España: Ediciones B.

BABICH, B. (2013): «O, Superman! Or being Towards Transhumanism: Martin Heidegger, Günther Anders, and Media Aesthetics», Divinatio (Studia Culturologica), 36, 41-99.

BEAR, G. (1998): Foundation and Chaos, EE.UU.: Harper Prism.

BENFORD, G. (1997): Foundation's Fear, EE.UU.: Harper Prism.

Bostrom, N. (2005): «A History of Transhumanist Thought», Journal of Evolution and Technology, 14(1), 1-25.

- (2011): «Una historia del pensamiento transhumanista», Argumentos de Razón Técnica, 14, 157-191.

BRIN, D. (1999): Foundation's Triumph, EE.UU.: Harper Prism.

BuRdeTt, M.S. Eschatology and the Technological Future (Routledge Studies in Religion), Nueva York: Taylor and Francis. Edición de Kindle.

Catalan, Z. (2010): «Aliens, Predators and Global Issues: The Evolution of a Narrative Formula», Cultura, Lenguaje y Representación, 8, 43-55.

Clarke, A. C., (1953): Childhood's End, Reino Unido de la Gran Bretaña, Ballantine Books.

Cuadros Contreras, R. (2008): «Reflexiones sobre alteridad y técnica: la figura del robot humanoide en algunas transposiciones de la literatura al cine», Revista CS, 2, 247-263.

DuARTe, M. (2010): «Transhumanismo», Revista de Humanidades Sarasuati, 6, 55-57.

Dvorsky, G.; Hughes, J. (2008): «Postgenderism: Beyond the Gender Binary», IEET Monograph Series. Disponible en https://ieet.org/archive/IEET-03-PostGender.pdf 
Fernández Ferres, J.; Pujal Carrera, M. (1996): Iniciación a la física, tomo I, España: Reverté.

González Melado, F. J.; Martínez Guisasola, J. M. (2012): «Hijos de un mismo Dios: ideología de género y transhumanismo». En ANDRADES LEDO, F. J. et al. (coords.): Razones para vivir y razones para esperar: homenaje al prof. D. José Román Flecha Andrés, Salamanca: Universidad Pontificia de Salamanca.

KingSbury, D. (2001): Psychohistorical Crisis, Ee.uU.: Tor Books.

Marín, F. R. (2012): «Ser o no ser [Cyborg]. Tecnología y cultura de la tradición prometeica a la tradición fáustica», Revista Trilogía, 7, 33-58.

Sandu, A.; Caras, A. (2013): «(Christian) Bioethical Dilemmas in Using Synthetic Biology and Nanotechnologies», Journal for the Study of Religions and Ideologies, 12(35), 158-177.

Suvin, D. (1984): Metamorfosis de la ciencia ficción, España: Fondo de Cultura Económica de España.

Tirosh-Samuelson, H. (2007): «Facing the Challenges of Transhumanism: Philosophical, Religious, and Ethical Considerations». Ensayo basado en la conferencia impartida en la conferencia anual del Instituto Metanexus sobre «Transdisciplinarity and the Unity of Knowledge: Beyond Science and Religion Dialogue». Disponible en http://www.metanexus.net/essay/ facing-challenges-transhumanism-philosophical-religious-and-ethical-considerations

Turchin, P. (2008): «Arise Cliodynamics», Nature, 454, 34-35.

- (2011): «Toward Cliodynamics - an Analytical, Predictive Science of History», Cliodynamics: The Journal of Quantitative History and Cultural Evolution, 2(1), 167-186.

VINGE, V. (1984): The Peace War, EE.UU.: Bluejay Books.

- (1993): «The Coming Technological Singularity», conferencia dictada durante VISION-21 Symposium, NASA Lewis Research Center y the Ohio Aerospace Institute, 30 y 31 de marzo de 1993. Disponible en https://ntrs.nasa.gov/archive/nasa/casi.ntrs.nasa.gov/19940022855.pdf 